\title{
La philosophie, le projet et le laboratoire
}

Les SHS dans l'enseignement secondaire italien

Philosophy, the project and the laboratory. Human and social sciences in Italian secondary education

La filosofía, el proyecto y el laboratorio. Las ciencias humanas y sociales en la enseñanza secundaria italiana

\section{Anita Gramigna}

Traducteur : Fiorenza Donella et Michel Lussault

\section{CpenEdition}

\section{Journals}

Édition électronique

URL : http://journals.openedition.org/ries/566

DOI : $10.4000 /$ ries.566

ISSN : 2261-4265

Éditeur

Centre international d'études pédagogiques

Édition imprimée

Date de publication : 1 décembre 2008

Pagination : 61-70

ISSN : 1254-4590

Référence électronique

Anita Gramigna, «La philosophie, le projet et le laboratoire », Revue internationale d'éducation de Sèvres [En ligne], 49 | décembre 2008, mis en ligne le 01 décembre 2011, consulté le 03 mai 2019. URL : http://journals.openedition.org/ries/566 ; DOI : 10.4000/ries.566 


\section{La philosophie, le projet et le laboratoire}

\section{Les SHS dans l'enseignement secondaire italien*}

\section{Anita Gramigna}

L'enseignement secondaire italien connaît, encore, aujourd'hui une séparation assez nette entre enseignement général et enseignement technique et professionnel, malgré les réformes réalisées pendant la dernière décennie. Une longue tradition, qui remonte à l'époque fasciste et à la réforme scolaire Gentile en 1923, a créé cette différence en instituant le lycée classique ${ }^{1}$ comme élément central de la formation secondaire, entre 11 et 18 ans, avec des critères d'accès très sélectifs pour en garantir le sérieux et mettre ainsi un frein à la mobilité sociale. Gramsci avait bien remarqué les défauts de cette réforme : institution d'une formation générale humaniste réservée aux enfants de la classe dominante et d'une formation professionnelle destinée aux enfants des classes subalternes. Il en précisait aussi le contexte : "le développement de la base industrielle, tant urbaine qu'agricole ", entraînant une "scission de l'enseignement en classique et technique (professionnel, mais non manuel), qui a remis en question le principe d'une formation secondaire orientée vers la culture générale, comme le voulait l'héritage de la tradition classique ${ }^{2}$. Confronté à la séparation irréductible culture générale/culture technique, Gramsci avait soutenu vers 1920 la nécessité de revenir à une formation générale humaniste unique pour tous les élèves âgés de six à seize ans, suivie par une phase de professionnalisation dans les trois dernières années de l'enseignement secondaire.

\section{UNE SÉPARATION CULTURELLEMENT ENRACINÉE}

Ce bref rappel historique nous semble significatif pour deux raisons : parce que les cadres posés par la réforme fasciste de l'école secondaire ne sont véritablement abolis en Italie qu'en 1999, grâce à la loi sur l'autonomie des établissements scolaires expérimentée à partir de 1997; et parce que, culturellement, ce

\footnotetext{
* Article traduit par Fiorenza Donella avec la collaboration de Michel Lussault.

1. Ndt : Après l'école élémentaire (cinq ans), le lycée classique prévoyait cinq années de gymnase, qui étaient la préparation pour accéder aux trois années de lycée classique. L'étude du latin et du grec était prévue pour tout le cursus. Actuellement, après le cycle primaire (cinq ans), le cycle secondaire comprend trois années de scuola media inferiore et cinq années de scuola media superiore pour les lycées généraux et pour les instituts techniques. Les instituts professionnels prévoient une formation de trois ans, complétée de deux ans pour l'obtention du baccalauréat professionnel.
}

2. Gramsci, 1975, p. 483 . 
sont deux intellectuels se référant à deux traditions philosophiques opposées, le matérialisme historique, dont Gramsci fut l'un des interprètes les plus originaux, et l'idéalisme, que Gentile réélabora dans son «actualisme», qui se sont particulièrement intéressés au sort du système scolaire italien.

Depuis les années 1920, la philosophie semble être le savoir capable d'offrir une véritable qualification et une vraie orientation intellectuelle : c'est la discipline qui caractérise, avec la langue et culture latine, la spécificité du lycée général comme filière réservée aux "élites», tandis que les autres disciplines, comme les mathématiques, les sciences, les lettres et les langues étrangères, sont enseignées dans toutes les filières du cycle secondaire, générales, techniques ou professionnelles, selon les programmes spécifiques prévus pour chaque filière.

Ce n'est donc pas un hasard si le gouvernement de centre-droit a proposé pendant la législature 2001-2006 une loi visant à la licealizzazione ${ }^{3}$ de la formation secondaire, avec l'insertion de l'enseignement de la philosophie dans toutes les filières technologiques, économiques, scientifiques. Cette généralisation avait été proposée aussi par le gouvernement de centre-gauche pendant la législature précédente, suivant l'avis autorisé d'experts reconnus.

Dans le même temps, les Régions se sont vu confier l'organisation de la formation professionnelle, ce qui a entraîné un déclassement pour les lycées professionnels qui ont ainsi perdu le statut d'établissements d'État. Cette décision repose sur l'idée d'une orientation précoce, le jeune devant envisager son avenir avant 14 ans, âge du premier choix, de la première «vocation». Implicitement, ce choix procède de la volonté de fournir au monde du travail une majorité de jeunes adaptés aux exigences des entreprises, ayant une culture générale réduite et donc plus faciles à utiliser en fonction des logiques du marché du travail : précarité, flexibilité, occupation intérimaire.

En principe, l'enseignement secondaire actuel en Italie est organisé pour permettre à l'élève de passer du lycée à la formation professionnelle et vice-versa, selon un parcours individuel qui prévoit, en tout cas, l'obligation de poursuivre la formation jusqu'à 18 ans. Mais ces deux modèles coexistent séparément et ne parviennent pas à s'harmoniser : d'un côté, on souligne la nécessité d'un savoir vaste, souple, ouvert aux transformations imposées par le monde du travail actuel, dans la perspective de la formation continue tout au long de la vie; de l'autre, on confirme la volonté de fournir aux élèves des compétences et des aptitudes spécifiques, qui constitueront la base certaine pour une future profession, surtout dans les trois dernières années du cursus.

Dans ce contexte la philosophie, plus encore que les sciences humaines, en particulier la psychologie et la sociologie, est le point de référence incontournable du premier modèle de formation secondaire. Il se propose de fournir à l'élève un savoir permettant d'effectuer de multiples tâches comme : ordonner

3. Ndlr : italianisme intraduisible : «lycéaliser» (donner le statut de lycée) à la formation secondaire. 
les connaissances venant de différentes disciplines, résoudre des problèmes, créer des rapports significatifs entre disciplines et stimuler des réflexions personnelles et constructives. En revanche, la psychologie et la sociologie pourraient entrer dans le deuxième modèle de formation, comme savoirs opératoires, importants aussi pour la formation professionnelle, par exemple pour faciliter les «relations publiques» ou les études statistiques, comme disciplines possédant des règles d'application certaines, en fonction d'une épistémologie linéaire et non complexe.

Pour comprendre la différence entre la philosophie et les autres sciences humaines, rappelons que dans le système de recrutement des professeurs en Italie, deux classes de concours sont proposées normalement : l'une pour les candidats venant de la faculté de philosophie et l'autre pour les candidats formés en psychologie, sociologie, pédagogie et, plus rarement, en philosophie. Le professeur de philosophie enseigne aussi l'histoire dans les lycées généraux, classiques et scientifiques. En revanche, le professeur de sciences humaines ou sociales dans les lycées techniques ou professionnels n'enseigne pas la philosophie, sauf si son poste relève d'un lycée pédagogique ou d'un lycée à orientation sociale issu d'une expérimentation.

\section{ENTRE HISTORICISME ET COMPLEXITÉ}

Toute la culture italienne, et non la seule philosophie, a été fortement influencée par l'idéalisme du philosophe Benedetto Croce. La diffusion de l'idéalisme a entraîné une sous-estimation de la portée théorique des sciences et une forte tendance à l'étude historique de l'ensemble des disciplines, remise en question seulement dans les années 1990, quand le besoin de réformer le secondaire a été ressenti comme une nécessité.

Cette empreinte historiciste, présente tant dans les manuels des élèves que dans la formation des professeurs, a été alors discutée en considérant que si l'étude de l'histoire de la philosophie permet à l'élève de situer les formes de la pensée dans leur contexte social, économique et culturel, en revanche elle ne l'aide pas à saisir le lien avec le présent et donc à apprendre à réfléchir, grâce à des critères philosophiques, sur les problèmes liés à l'actualité. Dans cette approche historico-philosophique ayant sa fin en elle-même, et de ce fait unilatérale, on perd la dimension épistémologique de la philosophie et on réduit son aspect logique qui permettrait une perspective critique. On perd surtout cette relation avec les autres disciplines, qui donne la possibilité de recomposer et d'intégrer ce qui a été artificiellement morcelé.

L'approche historique est encore aujourd'hui solidement ancrée dans l'enseignement de la philosophie. Elle domine dans les lycées généraux, situation provoquée par les carences de la loi de 1999 qui a donné l'autonomie aux établissements scolaires sans la compléter par une réforme des filières et, 
surtout, par une révision des programmes officiels propres à chaque filière. Sur ce point, qui revêt une grande importance stratégique, on en est resté au stade des conclusions des commissions d'experts, nommées tant par le gouvernement de centre-gauche (1996-2001) que par le gouvernement de centre-droit (20012006), dans le souci d'obtenir des avis significatifs pour pouvoir rechercher des convergences et un consensus sur les connaissances fondamentales dans l'apprentissage des jeunes.

Les philosophes nommés dans les commissions ont présenté des observations intéressantes sur l'importance actuelle de la philosophie, sur son rôle d'axe porteur dans le dialogue des différentes disciplines, dans la confrontation de différentes épistémologies. D’où la nécessité de faire découvrir aux élèves le caractère provisoire et hypothétique des connaissances, l'importance de construire un système ouvert, fonctionnant d'autant mieux que les élèves s'y insèrent dans un rapport de dialogue, sans préjugés ou hiérarchies de valeur préétablies. Les philosophes ont relevé aussi une tendance acritique dans l'enseignement des sciences, qui pousse les élèves à croire à la valeur absolue de ces connaissances.

Dans le cycle secondaire, c'est bien la philosophie qui devrait ouvrir la voie vers une culture de la complexité et de l'incertitude. La philosophie italienne, pour le cycle secondaire général, devrait chercher à mettre les jeunes en contact avec cette culture du doute, du relativisme et du possible, qui représente l'autre visage de la culture de notre siècle, pour qu'apprendre à apprendre ne reste pas seulement un slogan et que l'acquisition de méta-compétences commence à produire ses effets dans le système scolaire, avant d'être transférable dans le monde du travail du futur.

\section{LE PROJET ET LE LABORATOIRE}

Depuis la loi sur l'autonomie, tous les établissements sont tenus de proposer chaque année scolaire à leurs usagers, élèves et familles, un projet de l'offre de formation (POF, Piano dell'offerta formativa). La caractéristique principale du projet réside dans son lien à un territoire, dont l'établissement devient un élément vital, en synergie avec les institutions, les associations et les entreprises locales. Le POF, en plus des disciplines spécifiques de chaque filière prévues par les programmes officiels, propose aussi des projets d'innovation didactique, élaborés en fonction du contexte de l'établissement, afin de favoriser un apprentissage orienté vers l'actualité et vers l'environnement proche de l'élève, mais sans entraver les caractéristiques générales de toute forme de connaissance.

Les projets mettent souvent en relation des approches disciplinaires différentes autour d'un thème : par exemple, dans le delta du Po, le bassin a fait l'objet d'études historiques, géographiques, environnementales, économiques et touristiques, agricoles, productives, piscicoles, en fonction du type d'établis- 
sement scolaire et des finalités de sa formation. Cette pratique est intéressante parce qu'elle permet aux élèves de réaliser des activités d'apprentissage collaboratif ${ }^{4}$, d'effectuer des recherches sur le terrain ou de pratiquer des enquêtes en groupe, par exemple, avec des résultats significatifs en matière de qualité de leur formation.

Les POF présentent toutefois un risque : pérenniser les inégalités déjà existantes dans la structure de l'Italie. Indépendamment des financements réduits attribués par le ministère à chaque établissement scolaire pour les projets, les lycées peuvent avoir recours à d'autres financements, tant publics que privés : ainsi le Nord continue à être dans une position privilégiée par rapport au Sud, et la ville à être privilégiée par rapport à la campagne. Toutefois, pour beaucoup d'analystes, l'utilisation des POF est un pari à prendre parce que «les activités de projet produisent une transformation chez les élèves, si les professeurs respectent les caractères authentiques de l'épistémologie du projet, en valorisant leurs ressources humaines et en utilisant au mieux leur prédisposition innée pour la recherche ${ }^{5}$.

On valorise donc la perspective du «savoir faire», une praxis qui permet à l'élève de se rapprocher $\mathrm{du}$ monde du travail et de ses règles : apprendre à travailler en coopérant avec les autres pour la réalisation d'un but, s'approprier des stratégies d'observation et d'interprétation des données, concentrer son attention sur une situation déterminée et sur les éléments qui la distinguent, toutes ces activités aboutissent au développement de la compétence, si recherchée actuellement.

En plus de la notion de projet, une autre notion semble très importante, celle de «laboratoire». Plusieurs projets que nous avons consultés proposent aux élèves des activités pratiques significatives dans le domaine des sciences humaines : savoir utiliser avec une compétence croissante des méthodes comme l'interview, le questionnaire, l'autobiographie, dans un contexte déterminé et avec des personnes qui vivent dans une situation d'urgence. Par exemple, en utilisant la méthode de recherche des récits de vie, une classe d'un lycée pédagogique a essayé de reconstruire les moments les plus significatifs du vécu d'un groupe de personnes âgées résidant en maison de retraite. Cette expérience ne se limite pas à être un cas d'étude pratique original pour les élèves, mais les conduit à assumer une attitude éthique pour s’interroger sur le problème social posé par la vieillesse. En même temps, elle ouvre la voie vers d'autres méthodes et d'autres possibilités de recherche, vers une plus ample réflexion sur la variété des épistémologies qui caractérisent les différentes sciences.

Si l'autobiographie est une méthode typiquement «qualitative» qui tend à étudier la spécificité de chaque cas humain, les sciences naturelles,

\footnotetext{
4. Ndt: « cooperative learning».

5. Righetti, 2007, p. 68.
} 
habituellement, privilégient les enquêtes «quantitatives", les objets mesurables et tendent à expliquer les faits plutôt qu'à les interpréter. Il ne s'agit pas d'établir si une méthode est préférable à une autre, mais de comprendre que les disciplines peuvent choisir différents parcours de recherche en fonction des buts de leur analyse, sans pour cela faire de discriminations entre un modèle et un autre. Expérimenter une ou plusieurs activités de «laboratoire», peut permettre à l'élève de se poser des questions sur la nature et les finalités des différentes disciplines et de s'orienter, sur le plan personnel, en évaluant ses propres intérêts et ses propres «talents".

L'idée fondamentale, que l'on peut saisir dans les pratiques réelles des enseignants, est qu'aucun savoir ne peut être construit sans activité de "laboratoire». Ainsi, les sciences humaines se présentent aux yeux des apprenants seulement dans leur dimension d'application : il est vain, en effet, de théoriser sur les procédures de l'autobiographie, de parler de théories psychologiques ou de modèles ethnographiques, sans considérer la façon dont les chercheurs du secteur les appliquent dans leurs recherches sur le terrain, et sans travailler sur des exemples concrets. C'est pourquoi, lorsqu'on veut réaliser une enquête sur la condition de groupes marginalisés (par leur ethnie, leur situation économique, sociale, ou des problèmes de déviance), activité très répandue dans les lycées sociaux et pédagogiques, il s'avère très formateur de mettre en place des techniques de jeux de rôle. Ainsi, l'élève ne restera pas simple observateur d'un phénomène, mais saura se mettre à la place des personnes observées. Et, dans le futur, quand il travaillera, par exemple, dans le domaine de l'assistance sociosanitaire, l'élève qui aura ce type de formation pourra s'intéresser véritablement aux besoins des autres et ne répondra pas aux situations d'urgence par l'optique froide du bureaucrate, qui réduit les individus à des données statistiques.

\section{UNE EXPÉRIMENTATION SANS FIN?}

Le retard que le pouvoir législatif italien a pris pour lancer la «grande réforme» de l'enseignement secondaire, par ailleurs restée inaccomplie, a produit un phénomène intéressant d'adaptation «de la base» face aux mutations productives, sociales et culturelles de notre société. Ce phénomène vaste et varié, appelé ici "expérimentation», a produit des effets positifs mais n'a pas manqué d'avoir aussi des répercussions négatives. Cette expérimentation a-t-elle contribué à abréger ou à allonger le temps nécessaire à la réforme?

Malheureusement elle l'a augmenté, fournissant un alibi au vide législatif et aux tactiques des partis politiques, incapables de trouver de larges ententes, même dans un domaine d'intérêt public si important. À partir des années quatre-vingts s'est ainsi créé un mouvement d'opinion favorable à l'instauration d'une quantité incalculable de petites réformes, confiées à la responsabilité de chaque établissement, qui ont eu de ce fait le rôle d'espaces 
privilégiés pour tester des innovations dans les systèmes de formation, les méthodologies, les emplois du temps, les programmations, les objectifs. Or, en schématisant, le concept noble d'expérimentation à l'origine des sciences modernes se caractérise par le cycle observation-hypothèse-théorisation-vérification et ne doit pas être confondu avec le banal «faire une expérience» du sens commun.

La tendance dans le système scolaire italien a été malheureusement de retenir cette dernière définition et elle s'est diffusée, de manière hétérogène, sur le territoire national. La pratique généralisée du «bricolage avec les moyens du bord», a produit alors un mélange explosif avec la mode de la concurrence, comme si les établissements étaient des entreprises en compétition sur un marché. L'offre est devenue toujours plus déconcertante et incompréhensible pour les élèves et leurs familles, tout en vantant des facilités pour l'insertion dans le monde du travail, des modernisations stupéfiantes des cycles de formation, des simplifications des processus d'apprentissage grâce à des technologies extraordinaires. Les établissements ont commencé une guerre intestine, à coups de slogans publicitaires retentissants, tolérés quand ils n'étaient pas encouragés par le ministère de l'instruction publique et par ses antennes locales. Cependant, il faut rappeler que s'il n'y avait pas eu l'expérimentation, l'introduction de l'informatique dans les programmes scolaires aurait pris du retard et que les études linguistiques seraient restées l'apanage, plus ou moins exclusif, des classes privilégiées ${ }^{6}$.

En 1992, la commission Brocca, du nom du sous-secrétaire qui a coordonné les travaux au parlement, a tenté de remettre de la cohérence, et pour cela d'imposer le recours à un "espace de projet», initiative qui a eu des résultats appréciables. En effet, les équipes enseignantes sont depuis obligées de proposer des projets pédagogiques dans la programmation annuelle d'activités pour la classe, dans un esprit de collaboration interdisciplinaire. Ces projets doivent avoir une durée de deux ans, sur les trois années du cycle terminal, et prévoir un nombre d'heures suffisant, en fonction de ces indications : «en tout cas les projets orientés vers un sujet théorique doivent en partie prévoir un aspect opératoire, tandis que ceux qui sont orientés vers un problème pratique doivent être insérés dans un contexte culturel significatif ${ }^{7}$.

Les projets doivent donc sauvegarder la double exigence, théorique et pratique, et parvenir à mettre en évidence un nœud problématique qui puisse être analysé sous plusieurs points de vue. La classe doit être amenée à le percevoir comme un élément significatif à soumettre à une activité de recherche

6. Le lycée linguistique (cinq ans), à dominante langues modernes (étude de trois langues étrangères dans les trois dernières années du cycle et de deux langues étrangères pendant les deux premières années), a été à sa naissance un lycée privé en Italie. Avec les expérimentations, dans les années 1990, les lycées classiques ou les instituts techniques commerciaux ont pu créer des filières expérimentales à dominantes langues modernes.

7. Piani di studio della scuola secondaria superiore e programmi dei trienni, 1992, pp. 44-45. 
parce qu'il suscite curiosité, intérêt, désir d'approfondissement. On introduit ainsi la recherche-action comme stratégie «qui prend son mouvement de l'intérieur des situations pour repérer les problèmes, les procédés, les modèles, les langages, les technologies, les analyses qui amènent in itinere à la conscience épistémologique et à la reconnaissance des identités des disciplines, ainsi qu'à la résolution de problèmes ${ }^{8}$.

Aujourd'hui, en régime d'autonomie, chaque équipe pédagogique peut expérimenter des projets d'innovation didactique, en interdisciplinarité et par enseignement en tandem, sur des thèmes de grand intérêt, par exemple la bioéthique du point de vue philosophique, juridique, religieux, biologique, économique. Chaque professeur peut décider d'y participer pour enrichir son programme : il dispose d'un temps correspondant à $15-20 \%$ du nombre total d'heures disponibles dans l'année scolaire. Malheureusement, un risque de dérive existe quand, sur la base de variations minimes dans l'emploi du temps, par exemple une heure hebdomadaire d'éducation physique, un établissement scolaire peut se présenter indûment comme «lycée du sport», bien évidemment inexistant. Ou bien quand une heure supplémentaire d'histoire de l'art permet de promouvoir auprès des usagers un «lycée à orientation architecturale ", aussi peu avéré que le premier.

Ces pratiques d'information quasi mensongères dénaturent le sens authentique de l'autonomie, qui devrait permettre d'aller vers une formation plus sérieuse et produisent l'illusion du «pays des jouets», lieu fabuleux, justement, que Pinocchio voulait rejoindre : un élève à ne pas imiter.

\section{DANS LE LABYRINTHE UNIVERSITAIRE : LA DIFFICULTÉ DE CHOISIR}

La législation sur l'autonomie des établissements scolaires et, en général, des institutions de formation, a fini par produire des effets importants sur l'organisation de l'Université italienne. En effet, dans cette dernière décennie, chaque université a pu élargir l'offre de filières, ceci en l'absence d'un centre de coordination national ou d'un programme établi. Le goût bien italien $\mathrm{du}$ particularisme local (le pays "aux cents villes et aux mille clochers») ainsi que le culte de la différence par l'antagonisme ( «si mon ennemi est guelfe, je ne peux être que gibelin»), ont renforcé cette tendance. Des dénominations originales, peu communes, ont été attribuées aux filières traditionnelles pour répondre à l'exigence de nouveauté et pour faire augmenter, vraisemblablement, le nombre d'inscrits, notamment dans le domaine des sciences humaines. Ainsi, le pouvoir décisionnel local s'est renforcé et les universités ont suivi, comme l'avaient déjà fait les lycées, la logique qui consiste à drainer la demande

8. Ib. p. 46 . 
étudiante en usant s'il le faut du battage publicitaire. L'augmentation du nombre de filières a été déterminée aussi par la réorganisation des cursus de formation, puisque l'Italie s'est conformée au processus dit de Bologne. On a ainsi introduit le cursus licence, master, doctorat. Les pouvoirs politiques ont soutenu cette tendance. L'offre de filières s'est élargie, mais les étudiants se sont trouvés désorientés et le système n'a pas obtenu les résultats escomptés. Le nombre de diplômés n'a pas augmenté, la qualité des études ne s'est pas améliorée : le nombre d'étudiants abandonnant leurs études ainsi que le nombre d'étudiants au cursus irrégulier reste élevé en Italie, sans comparaison avec d'autres pays de l'OCDE.

Les activités d'orientation durant les deux dernières années de lycée confirment la difficulté rencontrée par les élèves dans leurs choix, ceci pour de nombreuses raisons, parmi lesquelles, au premier chef, la difficulté de comprendre la spécificité d'un cursus par rapport à un autre, à cause des variantes dans les dénominations de filières, pourtant en réalité proches, offertes par de nombreuses universités en situation de concurrence. Quels approfondissements pédagogiques spécifiques font-ils la différence entre le parcours d'un «animateur culturel» par rapport à celui d'un «éducateur professionnel extrascolaire»? Ces difficultés se manifestent par une réduction spectaculaire d'inscriptions dans des disciplines clés importantes dans la société, et qui, en Italie, possèdent une tradition illustre : les mathématiques en sont une bonne illustration.

La réduction d'inscriptions en mathématiques depuis quelques années a soulevé un débat non seulement parmi les experts, mais aussi dans le monde de la culture. L'explication «savante» la plus répandue, fait appel à un syllogisme simple, que nous pourrions synthétiser ainsi : a) les jeunes sont habitués à une vie facile; b) les mathématiques ne sont pas faciles; c) les jeunes préfèrent donc une vie sans mathématiques. Cette explication peut contenir une partie de vérité, mais elle nous semble quand même incomplète. Un autre élément expliquerait la désaffection : l'idée des mathématiques comme science exacte, qui semble immuablement peser, telle une chape de plomb, sur les facultés scientifiques traditionnelles : une seule science existe, dont les canons ont été établis définitivement, et en dehors de laquelle personne ne peut s'arroger le droit de se considérer comme un «scientifique». Ainsi, le savoir mathématique qui supporte les sciences de la nature est indiscutable et doit être administré à l'apprenant comme un médicament.

Les sciences exactes, elles aussi en crise, semblent dans l'Université italienne conserver intacte une patine épistémologique datant du XIX $\mathrm{X}^{\mathrm{e}}$ siècle, sauf exceptions. La révolution épistémologique du $\mathrm{XX}^{\mathrm{e}}$ siècle semble avoir davantage touché le secteur philosophique, qui produit des débats vivants et se différencie, produisant chez les jeunes diplômés une facilité d'adaptation aux différents segments du travail intellectuel d'aujourd'hui. En revanche, des 
sciences humaines comme la psychologie et la sociologie tiennent une position plus ambiguë : elles s'orientent davantage vers la technocratie, les études quantitatives et statistiques, et sont donc plus adaptées aux ambitions du modèle économique dominant, même si elles risquent alors de fournir un alibi culturel au modèle de globalisation actuel, pour lequel semble se réaliser, tristement, la prophétie de Pasolini : un développement sans progrès.

\section{Biblographie}

GRAMSCI A. (1975): Quadernidelcarcere. Torino, Einaudi.

REALE G. in Le conoscenzefondamentali per l'apprendimento dei giovaninellascuolaitaliananeiprossimidecenni. I materialidellaCommissione dei saggi (intervento dell'8 aprile 1997), Studi e Documenti degli Annali della Pubblica Istruzione, Roma, 1997.

RIGHETTI M. (2007): Organizzazione e progettazioneformativa. Milano, Angeli.

La cultura scientifica e tecnologica nel rinnovamento dell'istruzionetecnica. Studi e Documenti degli Annali della Pubblica Istruzione. Firenze, Le Monnier, 2000.

Piani di studio della scuola secondaria superiore e programmi dei trienni. Le proposte della Commissione Brocca. Studi e Documenti degli Annali della Pubblica Istruzione Firenze, Le Monnier, 1992. 